\title{
Prescribing Pattern of Analgesic Drugs at Boru Meda Hospital, North East, Amhara, Ethiopia
}

\author{
Abebaw Tegegne ${ }^{1 *}$, Wondesen Gashaw ${ }^{2}$, Dawit Kidane ${ }^{3}$ \\ ${ }^{1}$ Pharmacy Department, Dessie Health Science College, Amhara, Ethiopia \\ ${ }^{2}$ Pharmacy Department, Boru Meda Primary Hospital, Amhara, Ethiopia \\ ${ }^{3}$ Boru Meda Primary Hospital, Amhara, Ethiopia \\ Email: *mabebaw12@gmail.com
}

How to cite this paper: Tegegne, A., Gashaw, W. and Kidane, D. (2017) Prescribing Pattern of Analgesic Drugs at Boru Meda Hospital, North East, Amhara, Ethiopia. Pain Studies and Treatment, 5, 37-43. https://doi.org/10.4236/pst.2017.54004

Received: September 15, 2017

Accepted: October 27, 2017

Published: October 30, 2017

Copyright (c) 2017 by authors and Scientific Research Publishing Inc. This work is licensed under the Creative Commons Attribution International License (CC BY 4.0).

http://creativecommons.org/licenses/by/4.0/ (c) (i) Open Access

\begin{abstract}
Introduction: Pain is the sensory and emotional experience, which altered human health and well-being. When pain does not resolve, it may be associated with a serious disease, condition, or injury that needs timely medical care and also irrational prescribing of analgesics, which is possible to lead to unwanted side effects. Therefore, this study has been intended to evaluate the prescribing pattern of analgesics drugs at Boru Meda Hospital. Method: A retrospective cross sectional descriptive study was conducted. A total of 200 prescriptions containing analgesic were systematically collected by using well designed and pretested Data collection format. The necessary information was extracted from the prescription sheets by trained pharmacists and later analyzed using SPSS version 20 software. Result: Regarding prescribed analgesic acetaminophen 115 (36.9\%) was the most prescribed, and then followed diclofenac 83 (26.6\%) and Ibuprofen 64 (20.5\%). The maximum number of analgesic drugs was administered to patients by oral rout (75\%), which is followed by parenteral (19\%). About dosing approach $94 \%$ acetaminophen prescribed as needed (PRN) based, whereas ibuprofen $59.4 \%$ and tramadol $40 \%$ were prescribed as twice a day (bid) based. Acute fibril illness (13.6\%) was the highest reason for prescribing analgesics. Conclusion: Majority of the prescription analgesics drugs were prescribed as PRN dosing approaches with a very large percentage of oral medications. Some of the prescriptions revealed irrational prescribing of analgesics, in accordance of clinical indication, frequency of administration and combination of analgesic.
\end{abstract}

\section{Keywords}

Analgesics, Boru Meda Hospital, Opioids, NSAIDS 


\section{Introduction}

Pain is one of the most common complaints, which is unpleasant emotional and sensory experience associated with actual or potential tissue damage [1] [2]. Pharmacological approaches are using non opioid and opioid analgesics drugs by decreasing the generation of the mediators of pain at the site of tissue damage and also acting at higher brain centers, controlling the affective components of the pain [3]. Analgesics are defined as the drugs that relieve pain without blocking nerve impulse conduction or markedly altering sensory function [4]. Non-steroidal anti-inflammatory drugs (NSAIDs) are commonly prescribed group of analgesics drugs in clinical practice for the management of acute and moderate pain and inflammation [5].

The global importance of Rational use of medicines (RUM) is a great issue, which evaluating the accurate diagnosis, rational prescribing practice of prescribers and the pharmacist should ensure that the right drugs reach the right patient in prescribed dosage and quantity, with clear instructions on its appropriate use [6] [7].

The aim of this study is to obtain information regarding the prescribing pattern of analgesic by the prescribers in the inpatient departments (IPD) and outpatient departments (OPD) of Boru Meda Hospital (BMH).

\section{Methods}

A retrospective cross sectional descriptive study was conducted at IPD and OPD of BMH, North East Amhara, Dessie, Ethiopia, 2017. The prescriptions contain opioid and non opioid analgesic drugs were collected from patients attending the OPD and IPD. A total of 200 prescriptions containing opioid and non opioid analgesic (100 from each OPD and IPD) were collected. Data collection format was designed and trained pharmacists at the pharmacy department recorded the required data and information. The information extracted from the prescription sheets, later analyzed for information like-type of analgesic and its recurrence, rout of administration, frequency of administration, clinical indications for which analgesic were prescribed, pharmacological classes of analgesic prescribed.

Data generated from the questionnaire were analyzed using SPSS version 20 software after manual data verification and cleaning. Statistical analysis was not done due to it was a descriptive study.

\section{Ethical Consideration}

The study was approved by chief executive officer, and the head of the pharmacy department in the BMH. A formal letter was written from $\mathrm{BMH}$ and given to pharmacy department and also confidentiality of the information obtained from the patient's prescription reviewed throughout the study period was kept.

\section{Result}

During the study period, a total of 200 prescriptions prescribed to 111 females 
(55.5\%) and 89 males (44.5\%). The mean age of the patients included in the study was found to be $48.5 \pm 15.5$ years (Table 1 ).

Among 312 analgesic prescribed drugs both in the IPD and OPD acetaminophen 115 (36.9\%) was the most prescribed non opioid analgesic drug, then diclofenac 83 (26.6\%) and Ibuprofen 64 (20.5\%) were followed (Table 2).

As shown in Figure 1, for a total of 200 patients 312 Analgesic Drugs were prescribed, out of this 155 (50\%) were NSAIDs, followed by 115 (37\%) acetaminophen and 42 (13\%) Opioid Analgesic was accounted.

Table 1. Gender and age distribution frequency.

\begin{tabular}{cc}
\hline Variables & Frequency $\mathrm{n}(\%)$ \\
\hline Gender & \\
Male & $89(44.5 \%)$ \\
Female & $111(55.5 \%)$ \\
Age groups (year) & $48.5 \pm 14.5$ \\
\hline
\end{tabular}

Table 2. The percentage use of analgesics drugs.

\begin{tabular}{ccc}
\hline Drugs & Frequency & $\%$ \\
\hline Acetaminophen & 115 & 36.9 \\
Diclofenac & 83 & 26.6 \\
Ibuprofen & 64 & 20.5 \\
Tramadol & 40 & 12.8 \\
Indomethacin & 4 & 1.3 \\
Piroxicam & 4 & 1.3 \\
Pethidine & 2 & 0.6 \\
Aspirin & 0 & 0 \\
Morphine & 0 & 0 \\
Total & 312 & 100
\end{tabular}

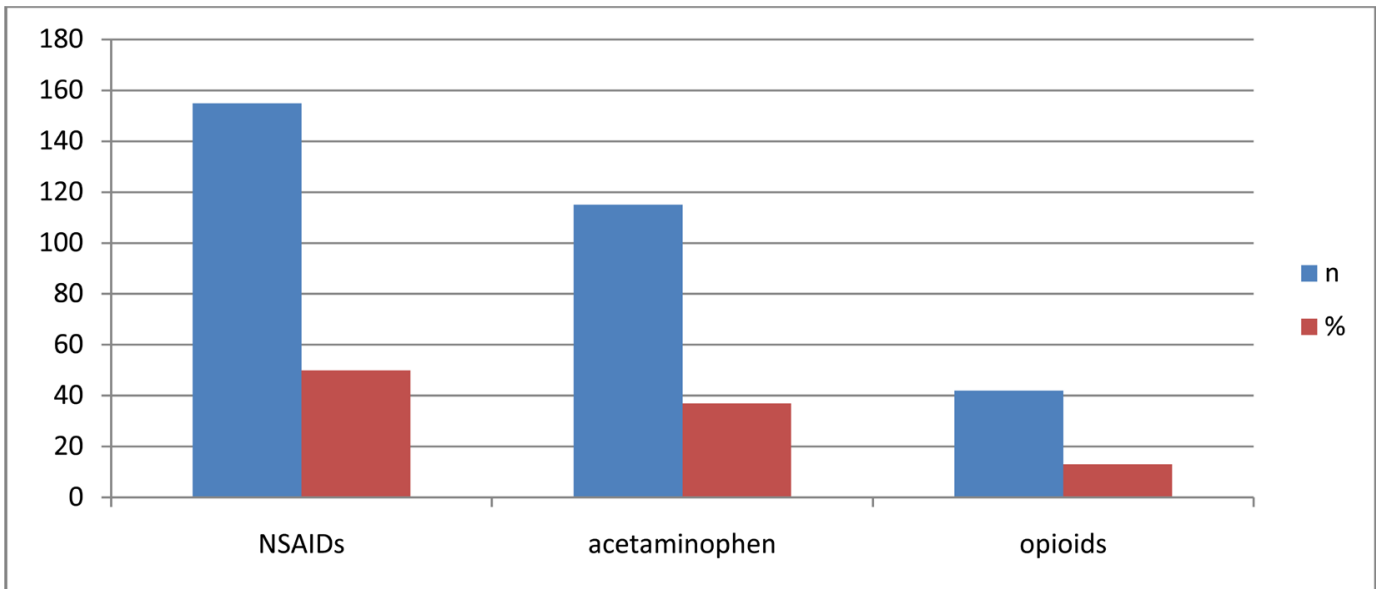

Figure 1. The proportion of prescribed class of analgesic drugs. 
As shown in Table 3, 94\% of acetaminophen, 34\% diclofenac, and 38\% (24) Ibuprofen were prescribed as PRN dosing approaches, whereas Ibuprofen $59.4 \%$, Tramadol $40 \%$ were prescribed as twice a day (bid) based. The highest percentage of Diclofenac injection 48\% (40) was administered at stat based.

The maximum number of analgesic drugs administered to patients by oral rout (75\%), followed parenteral (19\%) and rectal (6\%) drug administration rout (Figure 2).

The clinical indications for prescribing analgesics in this hospital were the highest prescribed for acute fibril illness and then followed by dyspepsia, cataract, pneumonia, and upper respiratory tract infection accounts more than half (53\%) (Table 4).

\section{Discussion}

Regarding this study, acetaminophen (37.6\%) was found the most prescribed drug; this may be showing minimal adverse effects with adequate efficacy as analgesic and antipyretic effect. According to the researches carried out in Nigeria, Bangladesh and Ghana Hospitals where indicated a similar result, that shows the largest percentage of prescribed analgesic drugs was acetaminophen [8] [9] [10]. However, other studies conducted in Sweden, Croatia and India showed that a controversial result, diclofenac was the most prescribed drug [11] [12] [13].

Table 3. Frequency of administration of analgesic drugs.

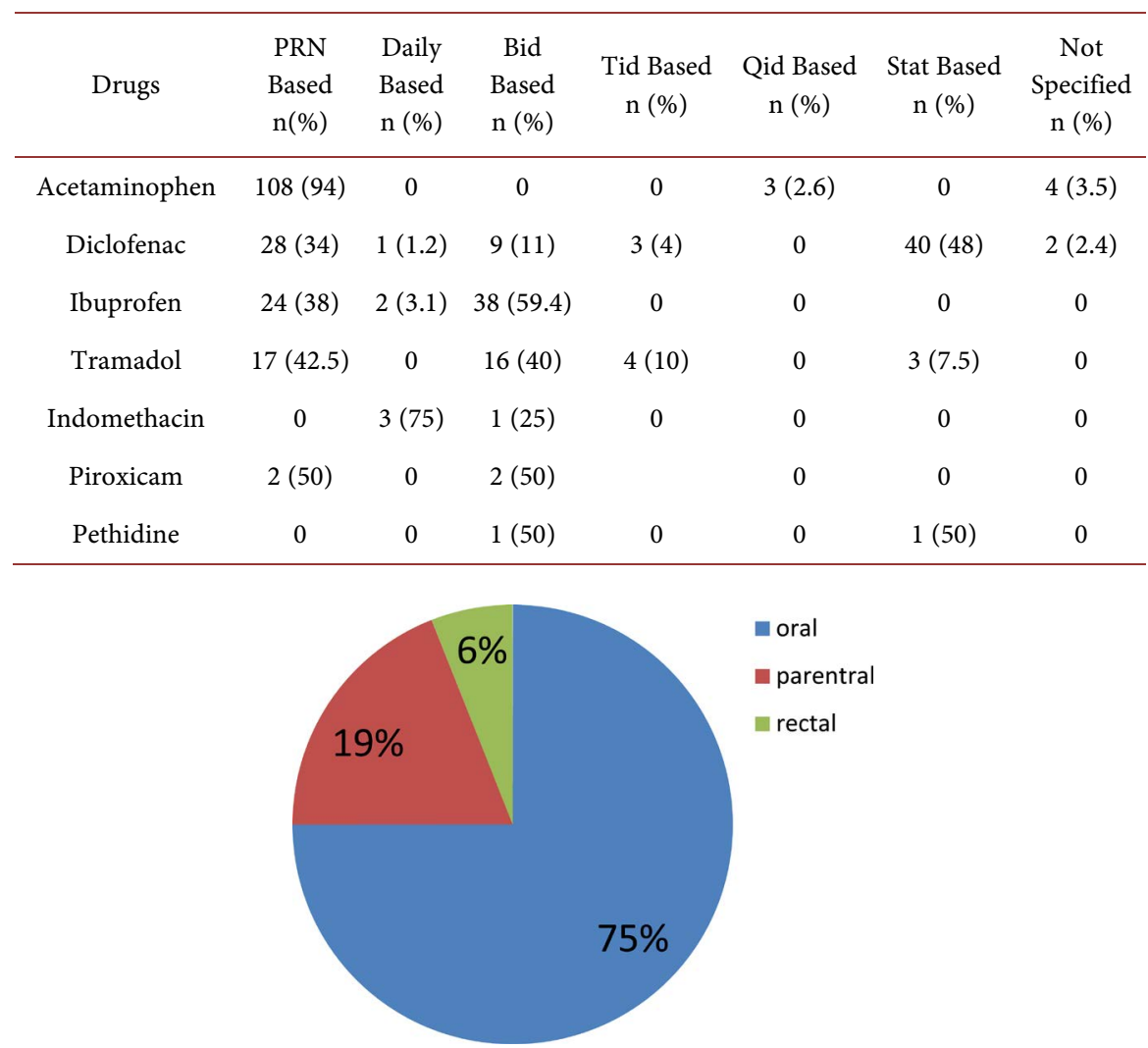

Figure 2. The percentage route of administration of analgesics. 
Table 4. Frequency of diagnosis for reason of prescribed analgesic.

\begin{tabular}{cccc}
\hline Diagnosis & Frequencyn (\%) & Diagnosis & Frequency n (\%) \\
\hline Acute fibril illness & $27(13.6)$ & Urinary tract infection & $7(3.5)$ \\
Dyspepsia & $26(13.1)$ & Fibro-myalgia & $6(3.0)$ \\
Cataract & $21(10.6)$ & Headache & $6(3.0)$ \\
Pneumonia & $18(9.0)$ & Post operation & $6(3.0)$ \\
Upper respiratory tract infection & $13(6.7)$ & typhoid fever & $5(2.5)$ \\
Rheumatoid arthritis & $10(5.0)$ & Muscle strain & $3(1.5)$ \\
Soft tissue injury & $10(5.0)$ & nephritic syndrome & $2(1.0)$ \\
Ulcer 2 ${ }^{\circ}$ to leprosy & $10(5.0)$ & Sepsis & $2(1.0)$ \\
Trauma & $9(4.5)$ & Corneal tear & $1(0.5)$ \\
Hypertension & $8(4.0)$ & Glaucoma & $1(0.5)$ \\
MDR TB & $7(3.5)$ & &
\end{tabular}

This study showed that NSAIDs were more prescribed analgesic drugs, out of this class diclofenac (26.6\%) and ibuprofen (20.5\%) more prescribed than indomethacin $(1.3 \%)$ and piroxicam (1.3\%). According to Rahul D., et al. study in India, ibuprofen was the most prescribed, followed by diclofenac [14]. The use of NSAIDs for the treatment of pain regarding in dental case, ibuprofen is an ideal prototype that were recommends for consideration in pain of dental origin [15].

Acetaminophen uses most of the time is irrational, it can cause serious liver problem even with less than maximum dose which is usually a $4 \mathrm{~g} /$ day. About 69\% patients were use Acetaminophen and NSAIDs dually and the dual user was mostly for treatment of arthritis pain, this reflects irrational prescribing practice [16].

The present study shows that Opioid analogues analgesic drugs like tramadol and pethidine were prescribed $12.8 \% \& 0.6 \%$ respectively (Table 1 ). The trend of prescribing strong opioids (like morphine, pethidine) was very low. This may be as a result of the misgiving of the issue of tolerance and addiction and/or these classes of drugs are reserved only for severe pain.

The present study findings were indicated that majority of analgesic drug prescribed as needed (prn) based frequency of administration, 94\% acetaminophen, $42.5 \%$ tramadol, 38\% ibuprofen, $34 \%$ diclofenac and 50\% piroxicam (Table 3). Non opioid analgesic taken on a regular schedule, not as prn based. Choosing of prn dosing is beneficial when rapid dose escalation or for patients who have rapidly decreasing analgesic requirements or intermittent pain separated by pain free intervals [3].

According to this study, $13.6 \%$ acute fibril illness was the most common clinical indication for analgesic drug prescribed, followed by $13.1 \%$ dyspepsia and $10.6 \%$ cataract. The matter of use of analgesia is the incidence of gastrointestinal (GI) bleeding side effects, specifically NSAID [17]. The safest drug in this regard has been noted as acetaminophen and Ibuprofen [18]. However, the overall NSAID 
therapy associated side effects has been related to the frequency and duration of treatments [19]. Administration of analgesic drug to relive pain related to dyspepsia indicates irrational use of medication.

\section{Conclusion}

Majority of the prescription analgesics drugs were prescribed as PRN dosing approaches with a very large percentage of oral medications. Some of the prescriptions revealed irrational prescribing of analgesics, in accordance of clinical indication, frequency of administration and combination of analgesic.

\section{References}

[1] Monfared, H., Sferra, J.J. and Mekhail, N. (2004) The Medical Management of Chronic Pain. Foot and Ankle Clinics, 9, 373-403. https://doi.org/10.1016/j.fcl.2004.02.002

[2] Tabish, A., Jha, R.K., Rathod, A.M., Rathod, R.M. and Gupta, K.K. (2012) Prescribing Trend of Analgesics in a Tertiary Health Care Setup of Rural Vidarbha. Research Journal of Pharmaceutical, Biological and Chemical Sciences, 3, 566-571.

[3] Craig, C.R. and Stitzel, R.E. (2014) Modern Pharmacology with Clinical Application. 5th Edition, Lippincott Williams \& Wilkins, Philadelphia, 634-640.

[4] Mohammed, T.C.H., Beegum, I.M. and Perumal, P. (2011) Prescribing Pattern of Analgesics in a Tertiary Care Hospital. International Journal of PharmTech Research, 3, 1521-1529.

[5] Rahman Md., S. Zinnat, A.B. and Samad Md., K. (2007) Prescribing Pattern of Non-Steroidal Anti-Inflammatory Drugs at Outpatient Departments of Teaching Hospitals. Bangladesh Journal of Pharmacology, 2, 1-6.

[6] Alam, K., Mishra, P., Prabhu, M., Shankar, P.R., Palaian, S., Bhandari, R.B. and Bista, D. (2006) A Study on Rational Drug Prescribing and Dispensing in Outpatients in a Tertiary Care Teaching Hospital of Western Nepal. Kathmandu University Medical Journal, 4, 436-443.

[7] Lea, F. (1985) Pharmacy Communication. In: Hassan, W.E., Ed., Hospital Pharmacy, 5th Edition, Lippincott Williams \& Wilkins, Philadelphia, 154-159.

[8] Abu-Saeed, K., Omuya, L.M., Buhari, A.-S.M., Saka, A.O. and Saka, M.J. (2012) Evaluation of Analgesics Usage in Pain Management among Physicians. Journal of Applied Pharmaceutical Science, 2, 194-198.

[9] Rahman, S., Begum, Z.A. and Samad, K. (2007) Prescribing Pattern of Nonsteroidal Anti-Inflammatory Drugs at Outpatient Departments of Teaching Hospitals. Bangladesh Journal of Pharmacology, 2, 1-6.

[10] Owusu-Ansah, S.A. (2009) Analgesic Use at the Polyclinic OPD of Komfo Anokye Teaching Hospital. Thesis, Kwame Nkrumah University of Science and Technology, Kumasi, 3-4.

[11] Rahul, D., Yasmin, G., Jaspreet, S.B. and Amandeep, S.A. (2015) Survey of Analgesic and Anti-Inflammatory Drug Prescription for Oral. Plastic and Aesthetic Research, 2, 51-55.

[12] Vlahovic-Palcevski, V., Wettermark, B. and Bergman, U. (2002) Quality of Nonsteroidal Anti-Inflammatory Drug Prescribing in Croatia (Rijeka) and Sweden (Stockholm). European Journal of Clinical Pharmacology, 58, 209-214. https://doi.org/10.1007/s00228-002-0449-2 
[13] Hayas Muhammed, T.C., MufidaBeegum, I. and Perumal, P. (2011) Prescribing Pattern of Analgesics in a Tertiary Care Hospital. International Journal of PharmTech Research, 3, 1521-1529.

[14] Kumarasingam, T., Revathy, S. and Mukherjee, D. (2015) Drug Utilization Pattern of Analgesics among Postoperative Patients in a Tertiary Care Hospital. Der Pharmacia Lettre, 6, 40-46.

[15] Becker, D.E. (2010) Pain Management: Part 1: Managing Acute and Postoperative Dental Pain. Anesthesia Progress, 57, 67-79. https://doi.org/10.2344/0003-3006-57.2.67

[16] Somia, G. and Maria, A. (2014) Prevalence of Prescribing Pattern of More than One NSAID in Pakistan. Journal of Scientific and Innovative Research, 3, 148-154.

[17] Mehlisch, D.R. (2002) The Efficacy of Combination Analgesic Therapy in Relieving Dental Pain. The Journal of the American Dental Association, 133, 861-871.

[18] Waring, W.S., Robinson, O.D., Stephen, A.F., Dow, M.A. and Pettie, J.M. (2008) Does the Patient History Predict Hepatotoxicity after Acute Paracetamol Overdose? QJM, 101, 121-125.

[19] Jackson, C.H., MacDonald, N.C. and Cornett, J.W. (1984) Acetaminophen: A Practical Pharmacologic Overview. Canadian Medical Association Journal, 131, 25-32. 